\title{
A relaxation method for calculating detonation in condensed explosives
}

\author{
Ming Yu \& Zhibo Ma \\ Institute of Applied Physics and Computational Mathematics, China
}

\begin{abstract}
Based on the relaxation approximation, the nonlinear governing equations of detonation in condensed explosives are transformed into linear relaxation systems, which can easily adopt simple and effective high resolution scheme. A fifth-order WENO reconstruction in space discretization and a fifth-order IMEX scheme of linear multistep methods with monotonicity and TVB in time discretization are utilized, where it can be avoid solving Riemann problem and calculating the Jacobian matrix of nonlinear flux, and it is not necessary to split the reaction source term. The present method is applied to simulate the steady structure of a one-dimensional planar detonation wave in condensed explosives, and the results demonstrate the excellent performance of the present method.

Keywords: relaxation method, detonation wave, condensed explosives, high resolution scheme.
\end{abstract}

\section{Introduction}

The design of complex engineering devices that use high explosives to do useful and controlled work requires the capability to exactly numerically simulate detonation. At present, the Lagrangian method is the most widely used to simulate detonation of condensed explosives. However, it is well known that the Lagrangian method has the following distinct shortcomings [1]: 1) to preserve with difficulty conservation of the total energy due to usually using staggered discretization grids; 2) to commonly smear the discontinuity of detonation wave due to using artificial viscosity, and give with difficulty the exact coefficient of artificial viscosity; 3) to be only one-order precision in temporal and spatial discretization, and extend with difficulty to high-order precision; 4) to easily distort discretization grids to result in calculation fail when using small grids. 
The Eulerian method with high-order precision and high resolution can well overcome the shortcomings of the Lagrangian method, so more and more attention to the Eulerian method has been paid. Representative works show some characteristic results of Eulerian method [2-5]: using a two-order Godunov scheme, adopting simple equation of state and chemical reaction model, applying split idea to process source term of chemical reaction law.

The governing equations of the detonation in condensed explosives are nonlinear hyperbolic conservation system with strongly stiff reaction source term and complex equation of state. The strong stiffness of reaction source term and the complexity of equation of state bring enormous difficulty to apply high-order precision and high resolution scheme.

When strongly stiff source term is discretized, insufficient spatial/temporal resolution may cause an incorrect propagation speed of discontinuities and nonphysical states for standard dissipative numerical methods that were developed for non-source flows. Yee et al. $[6,7]$ point out that the phenomenon of wrong propagation speed of discontinuities is connected with the smearing of the discontinuities caused by the discretization of the advection term. The smearing introduces a nonequilibrium state into the calculation, thus as soon as a nonequilibrium value is introduced in this manner, the source term turns on and immediately restores equilibrium, while at the same time shifting the discontinuity to a cell boundary. So, exactly capturing detonation wave discontinuity requires the high resolution scheme with low numerical dissipation. At present, the most high resolution schemes have utilized Riemann Solver based on simple equation of state, such as the perfect gas with Gamma law. However, unrected solid component and gas product component of detonation in condensed explosives usually utilize some complex equation of state, such as Jones-Wilkins-Lee (JWL), HOM, BKW, Davis, extremely, SESAME data library, also, the temperature of mixing zone in chemical reaction needs to iterative operation when generally considering pressure and temperature as equilibrium state. Apparently, the high resolution scheme based on Riemann Solver is difficult to numerically calculate the flow equations of detonation in condensed explosives. In order to exactly capturing the discontinuity, besides the high resolution in spatial discretization, the high resolution in temporal discretization is very necessary. Representative results [9] show that the unsplitting explicit and implicit scheme is much more reliable: the advection term adopts explicit scheme, and the source term adopts implicit scheme.

Recently developing relaxation method is a kind of effective strategy to numerically solve hyperbolic conservation system [10-13]. The main idea of the relaxation method is to transform the nonlinear hyperbolic conservation system into linear hyperbolic relaxation equations by means of relaxation approximation. When the relaxation rate tends to zero and the subcharacteristic condition is satisfied, the solution of the relaxation equations converges to the solution of the original hyperbolic conservation system. In comparison with upwind schemes such as the Godunov scheme, relaxation method does not require the Riemann Solver and the computation of its Jacobians. These features make the relaxation method particularly suitable for those systems where the Riemann problem is 
difficult to solve or when it is not possible to perform analytical expression for Jacobians. The relaxation method is gradually applied to gas dynamics [14], shallow water motion [15], dynamic phase transition [16], multicomponent flow [17], magnetohydrodynamic [18].

In the paper, the relaxation method is applied to numerically simulate the typical detonation problem about the condensed explosives. After the nonlinear governing equations of the condensed explosives are transformed into linear relaxation equations, an improved fifth-order weighted essentially nonoscillatory (WENO) [19] is utilized to spatially discretize and a fifth-order IMEX scheme of linear multistep methods with general monotonicity and boundedness properties is utilized to temporally discretize [9]. The numerical example about one-dimensional steady structure of detonation wave in PBX-9404 demonstrates that our method has high accuracy and high resolution properties.

\section{Governing equations of detonation in condensed explosives}

The one-dimensional flow equations of detonation in condensed explosives under Eulerian frame are following:

$$
\frac{\partial \boldsymbol{u}}{\partial t}+\frac{\partial \boldsymbol{f}(\boldsymbol{u})}{\partial r}=\boldsymbol{s}(\boldsymbol{u})
$$

where

$$
\begin{aligned}
& \boldsymbol{u}=\left[\rho r^{N}, \rho v r^{N}, \rho E r^{N}, \rho \lambda r^{N}\right]^{T}, \\
& \boldsymbol{f}(\boldsymbol{u})=\left[\rho v r^{N},\left(\rho v^{2}+p\right) r^{N},(\rho E+p) v r^{N}, \rho v \lambda r^{N}\right]^{T}, \\
& \boldsymbol{s}(\boldsymbol{u})=\left[0, N r^{N-1} p, 0, \rho r^{N} R(\rho, p, \lambda)\right]^{T},
\end{aligned}
$$

where $N$ is geometry factor $(N=0$ for plane, $N=1$ for cylinder, and $N=2$ for sphere), and $R$ is chemical reaction rate, where three-term Lee-Tarver reaction law is adopted [8]:

$$
R=I\left(\eta_{1}-1-a\right)^{n}(1-\lambda)^{y}+G_{1}(1-\lambda)^{y_{1}} \lambda^{x_{1}} p^{z_{1}}+G_{2}(1-\lambda)^{y_{2}} \lambda^{x_{2}} p^{z_{2}} .
$$

The unrected solid component and gas product component of detonation in condensed explosives utilize Jones-Wilkins-Lee equation of state. On assumption that the pressure and temperature in reaction mixing zone is equilibrium, the state of mixing zone may express as (subscript $\mathrm{s}$ denotes solid component and subscript $\mathrm{g}$ denotes gas product component): 


$$
\left\{\begin{array}{l}
V=(1-\lambda) V_{s}+\lambda V_{g} \\
e=(1-\lambda) e_{s}+\lambda e_{g} \\
p=A_{s} \exp \left(-R_{1 s} V_{s}\right)+B_{s} \exp \left(-R_{2 s} V_{s}\right)+\frac{\omega_{s} C_{V s}}{V_{s}} T \\
p=A_{g} \exp \left(-R_{1 g} V_{g}\right)+B_{g} \exp \left(-R_{2 g} V_{g}\right)+\frac{\omega_{g} C_{V g}}{V_{g}} T \\
e_{s}=\frac{A_{s}}{\rho_{0} R_{1 s}} \exp \left(-R_{1 s} V_{s}\right)+\frac{B_{s}}{\rho_{0} R_{2 s}} \exp \left(-R_{2 s} V_{s}\right)+\frac{C_{V s}}{\rho_{0}} T \\
e_{g}=\frac{A_{g}}{\rho_{0} R_{1 g}} \exp \left(-R_{1 g} V_{g}\right)+\frac{B_{g}}{\rho_{0} R_{2 g}} \exp \left(-R_{2 g} V_{g}\right)+\frac{C_{V g}}{\rho_{0}} T
\end{array},\right.
$$

where $V=\rho_{0} / \rho$ is the relative volume, $e$ is the internal energy per mass.

\section{Establishment of relaxation equations}

By means of relaxation approximation, the governing equations about condensed explosives may be replaced by the following relaxation system:

$$
\left\{\begin{array}{l}
\frac{\partial \boldsymbol{u}}{\partial t}+\frac{\partial \boldsymbol{w}}{\partial r}=\boldsymbol{s}(\boldsymbol{u}) \\
\frac{\partial \boldsymbol{w}}{\partial t}+A^{2} \frac{\partial \boldsymbol{u}}{\partial r}=\frac{\boldsymbol{f}(\boldsymbol{u})-\boldsymbol{w}}{\varepsilon}
\end{array}\right.
$$

where $\boldsymbol{w}$ is a middle variable, $\boldsymbol{A}=\operatorname{diag}\left[a_{1}, a_{2}, a_{3}, a_{4}\right]$ is a positive diagonal matrix, $0<\varepsilon \leq 1$ is a relaxation rate.

The linear character of relaxation system (2) is utilized to construct simple and effective high resolution scheme. Papers $[10,11]$ point out that the solutions of (2) approach the solutions of original problem (1): $\boldsymbol{w} \rightarrow \boldsymbol{f}(\boldsymbol{u})$, as $\varepsilon \rightarrow 0$, provided the following subcharacteristic condition holds:

$$
-a_{k} \leq \frac{\partial \boldsymbol{f}(\boldsymbol{u})}{\partial \boldsymbol{u}} \leq a_{k}(k=1,2,3,4) \text { for all } \boldsymbol{u} \text {. }
$$

The role of relaxation rate in numerical scheme may be analyzed [12].

Because $\boldsymbol{w}$ can converge to $\boldsymbol{f}(\boldsymbol{u})$, there is a Chapman-Enskog expansion:

$$
\boldsymbol{w}=\boldsymbol{f}(\boldsymbol{u})+\varepsilon \boldsymbol{f}_{1}(\boldsymbol{u})+\varepsilon^{2} \boldsymbol{f}_{2}(\boldsymbol{u})+\cdots
$$

By substituting (3) into (2) and collecting terms, a first-order approximation of system (2) can be determined: 


$$
\begin{aligned}
\frac{\partial \boldsymbol{u}}{\partial t}+\frac{\partial \boldsymbol{f}(\boldsymbol{u})}{\partial r} & =\boldsymbol{s}(\boldsymbol{u})+\varepsilon \frac{\partial}{\partial r}\left[\left(\frac{\partial \boldsymbol{f}(\boldsymbol{u})}{\partial \boldsymbol{u}}\right) \boldsymbol{s}(\boldsymbol{u})\right] \\
& +\varepsilon \frac{\partial}{\partial r}\left\{\left[\boldsymbol{A}^{2}-\left(\frac{\partial \boldsymbol{f}(\boldsymbol{u})}{\partial \boldsymbol{u}}\right)^{2}\right] \frac{\partial \boldsymbol{u}}{\partial r}\right\}
\end{aligned}
$$

Thus, system (4) is dissipative if the subcharacteristic condition holds. Introducing relaxation rate is equivalent to introducing numerical dissipation. In practice, the elements of diagonal matrix in system (2) may be chosen as: $\boldsymbol{A}=\max (|\partial \boldsymbol{f}(\boldsymbol{u}) / \partial \boldsymbol{u}|)$ in the whole flowfield zone. Thus, $\boldsymbol{A}$ is a constant matrix, and the bigger $\boldsymbol{A}$ implies the bigger numerical dissipation.

\section{Solution of relaxation equations}

Diagonalize the system (2) and holds:

$$
\left\{\begin{array}{l}
\frac{\partial(\boldsymbol{w}-\boldsymbol{A u})}{\partial t}-\boldsymbol{A} \frac{\partial(\boldsymbol{w}-\boldsymbol{A u})}{\partial r}=\frac{\boldsymbol{f}(\boldsymbol{u})-\boldsymbol{w}}{\varepsilon}-\boldsymbol{A s}(\boldsymbol{u}) \\
\frac{\partial(\boldsymbol{w}+\boldsymbol{A u})}{\partial t}+\boldsymbol{A} \frac{\partial(\boldsymbol{w}+\boldsymbol{A u})}{\partial r}=\frac{\boldsymbol{f}(\boldsymbol{u})-\boldsymbol{w}}{\varepsilon}+\boldsymbol{A s}(\boldsymbol{u})
\end{array}\right.
$$

It can be found that the system (5) has constant linear character with characteristic line $d r / d t= \pm \boldsymbol{A}$ and Riemann Invariables $\boldsymbol{w} \pm \boldsymbol{A} \boldsymbol{u}$.

A semi-discrete finite difference scheme with uniform space sizes for the system (5) can be approximated into:

$$
\left\{\begin{array}{l}
\frac{d \tilde{\boldsymbol{u}}_{i}}{d t}=\boldsymbol{A} \frac{\tilde{\boldsymbol{u}}_{i+1 / 2}-\tilde{\boldsymbol{u}}_{i-1 / 2}}{\Delta r}+\frac{1}{\varepsilon}\left[\boldsymbol{f}\left(\boldsymbol{A}^{-l} \frac{\tilde{\boldsymbol{w}}_{i}-\tilde{\boldsymbol{u}}_{i}}{2}\right)-\frac{\tilde{\boldsymbol{w}}_{i}+\tilde{\boldsymbol{u}}_{i}}{2}\right]-\boldsymbol{A} \boldsymbol{s}\left(\boldsymbol{A}^{-l} \frac{\tilde{\boldsymbol{w}}_{i}-\tilde{\boldsymbol{u}}_{i}}{2}\right) \\
\frac{d \tilde{\boldsymbol{w}}_{i}}{d t}=-\boldsymbol{A} \frac{\tilde{\boldsymbol{w}}_{i+1 / 2}-\tilde{\boldsymbol{w}}_{i-1 / 2}}{\Delta r}+\frac{1}{\varepsilon}\left[\boldsymbol{f}\left(\boldsymbol{A}^{-1} \frac{\tilde{\boldsymbol{w}}_{i}-\tilde{\boldsymbol{u}}_{i}}{2}\right)-\frac{\tilde{\boldsymbol{w}}_{i}+\tilde{\boldsymbol{u}}_{i}}{2}\right]+\boldsymbol{A} \boldsymbol{s}\left(\boldsymbol{A}^{-1} \frac{\tilde{\boldsymbol{w}}_{i}-\tilde{\boldsymbol{u}}_{i}}{2}\right)
\end{array}\right.
$$

where $\tilde{\boldsymbol{u}}=\boldsymbol{w}-\boldsymbol{A u}, \tilde{\boldsymbol{w}}=\boldsymbol{w}+\boldsymbol{A u}$.

The spatially discretizing terms $\tilde{\boldsymbol{u}}_{i \pm l / 2}$ and $\tilde{\boldsymbol{w}}_{i \pm l / 2}$ in system (6) may adopt an improved fifth-order weighted essentially non-oscillatory (WENO) [19]:

$$
\begin{gathered}
\tilde{\boldsymbol{u}}_{i+1 / 2}=w_{1} \tilde{\boldsymbol{u}}_{i+1 / 2}^{(1)}+w_{2} \tilde{\boldsymbol{u}}_{i+1 / 2}^{(2)}+w_{1} \tilde{\boldsymbol{u}}_{i+1 / 2}^{(2)}, \\
\tilde{\boldsymbol{u}}_{i+1 / 2}^{(1)}=\frac{1}{3} \tilde{\boldsymbol{u}}_{i-2}-\frac{7}{6} \tilde{\boldsymbol{u}}_{i-1}+\frac{11}{6} \tilde{\boldsymbol{u}}_{i}, \\
\tilde{\boldsymbol{u}}_{i+1 / 2}^{(2)}=-\frac{1}{6} \tilde{\boldsymbol{u}}_{i-1}+\frac{5}{6} \tilde{\boldsymbol{u}}_{i}+\frac{1}{3} \tilde{\boldsymbol{u}}_{i+1}, \\
\tilde{\boldsymbol{u}}_{i+1 / 2}^{(3)}=\frac{1}{3} \tilde{\boldsymbol{u}}_{i}+\frac{5}{6} \tilde{\boldsymbol{u}}_{i+1}-\frac{1}{6} \tilde{\boldsymbol{u}}_{i+2},
\end{gathered}
$$




$$
\begin{gathered}
w_{k}=\alpha_{k}^{*} /\left(\sum_{l=1}^{3} \alpha_{l}^{*}\right), \alpha_{k}^{*}=g_{k}\left(w_{k}^{(J S)}\right), \\
g_{k}(w)=\frac{w\left(\bar{w}_{k}+\bar{w}_{k}^{2}-3 \bar{w}_{k} w+w^{2}\right)}{\bar{w}_{k}^{2}+w\left(1-2 \bar{w}_{k}\right)}, \\
w_{k}^{(J S)}=\alpha_{k} /\left(\sum_{l=1}^{3} \alpha_{l}\right), \alpha_{k}=\frac{\bar{w}_{k}}{\left(\delta+\beta_{k}\right)^{2}}, \\
\beta_{1}=\frac{13}{12}\left(\tilde{\boldsymbol{u}}_{i-2}-2 \tilde{\boldsymbol{u}}_{i-1}+\tilde{\boldsymbol{u}}_{i}\right)^{2}+\frac{1}{4}\left(\tilde{\boldsymbol{u}}_{i-2}-4 \tilde{\boldsymbol{u}}_{i-1}+3 \tilde{\boldsymbol{u}}_{i}\right)^{2}, \\
\beta_{2}=\frac{13}{12}\left(\tilde{\boldsymbol{u}}_{i-1}-2 \tilde{\boldsymbol{u}}_{i}+\tilde{\boldsymbol{u}}_{i+1}\right)^{2}+\frac{1}{4}\left(\tilde{\boldsymbol{u}}_{i-1}-\tilde{\boldsymbol{u}}_{i+1}\right)^{2}, \\
\beta_{3}=\frac{13}{12}\left(\tilde{\boldsymbol{u}}_{i}-2 \tilde{\boldsymbol{u}}_{i+1}+\tilde{\boldsymbol{u}}_{i+2}\right)^{2}+\frac{1}{4}\left(3 \tilde{\boldsymbol{u}}_{i}-4 \tilde{\boldsymbol{u}}_{i+1}+\tilde{\boldsymbol{u}}_{i+2}\right)^{2} .
\end{gathered}
$$

While temporally discretizing the system (6), the following ordinary differential equations can be determined:

$$
\frac{d \boldsymbol{q}}{d t}=\mathcal{F}(\boldsymbol{q})+\mathcal{S}(\boldsymbol{q})
$$

where $\boldsymbol{q}=[\tilde{\boldsymbol{u}}, \tilde{\boldsymbol{w}}]^{T}, \mathcal{F}(\boldsymbol{q})$ denotes the discretization of the advection term in system (6), $\mathcal{S}(\boldsymbol{q})$ denotes the discretization of the source term in system (6).

A fifth-order IMEX scheme of linear multistep methods with general monotonicity and boundedness properties [9] is adopted to discretize the ordinary differential equations (7):

$$
\begin{aligned}
\boldsymbol{q}_{n} & =\frac{13553}{4096} \boldsymbol{q}_{n-1}-\frac{38121}{8192} \boldsymbol{q}_{n-2}+\frac{7315}{2048} \boldsymbol{q}_{n-3} \\
& -\frac{6161}{4096} \boldsymbol{q}_{n-4}+\frac{2264}{8192} \boldsymbol{q}_{n-5} \\
& +\frac{10306951}{5898240} \Delta t \mathcal{F}_{n-1}-\frac{13656497}{2949120} \Delta t \mathcal{F}_{n-2}+\frac{1249949}{245760} \Delta t \mathcal{F}_{n-3} \\
& -\frac{7937687}{2949120} \Delta t \mathcal{F}_{n-4}-\frac{3387361}{5898240} \Delta t \mathcal{F}_{n-5} \\
& +\frac{4007}{8192} \Delta t \mathcal{S}_{n}-\frac{4118249}{5898240} \Delta t \mathcal{S}_{n-1} \\
& +\frac{768703}{2949120} \Delta t \mathcal{S}_{n-2}+\frac{47849}{245760} \Delta t \mathcal{S}_{n-3} \\
& -\frac{725087}{2949120} \Delta t \mathcal{S}_{n-4}+\frac{502321}{5898240} \Delta t \mathcal{S}_{n-5}
\end{aligned}
$$

A three-order Runge-Kutta method is used for starting procedure of the IMEX scheme of linear multistep method. 
Finally, the relaxation scheme with temporal-spatial fifth-order precision about the detonation flows in condensed explosives turns into the expression (8). It is worthy of indicating that the discretization procedure does not solve Riemann problem.

\section{Numerical example}

In this section, the steady structure of one-dimensional planar detonation wave in condensed explosives PBX-9404 is calculated. The JWL parameters for unreacted solid component of PBX-9404 are $(g-c m-\mu s)$ [8]: $\rho_{0}=1.842$, $A_{s}=69.69, \quad B_{s}=-1.727, \quad R_{1 s}=7.8, \quad R_{2 s}=3.9, \quad \omega_{s}=0.8578$, $C_{V s}=2.505 \times 10^{-5}$, and the JWL parameters for gas product component of PBX9404 are: $A_{g}=8.524, \quad B_{g}=0.1802, \quad R_{1 g}=4.6, \quad R_{2 g}=1.3, \quad \omega_{g}=0.38$, $C_{V g}=1.0 \times 10^{-5}, E_{0}=0.102$; and the corresponding parameters of Lee-Tarver reaction law are: $I=7.43 \times 10^{11}, n=20.0, y=0.667, \quad G_{1}=3.1, \quad x_{1}=0.111$, $y_{1}=0.667, z_{1}=1.0, \mathrm{G}_{2}=400.0, \quad x_{2}=1.0, \quad y_{2}=0.333, z_{2}=2.0, \quad a=0.0$. The values for Von Neumann spike are: $p_{N}=0.563, V_{N}=0.6075, u_{N}=0.347$; and the corresponding values for Chapman-Jouguet state are: $p_{C J}=0.370$, $V_{C J}=0.7403, u_{C J}=0.229, \quad D=0.8809$.

The relaxation rate takes $\varepsilon=10^{-7}$.

When the detonation arrives at the steady state, the distribution of physical variables in chemical reaction zone can be exactly obtained by means of the Hugoniot relations of detonation wave. In this example, the calculating length of explosives takes $4.0 \mathrm{~cm}$, and the explosives is initiated by the Chapman-Jouguet condition at its left hand. The distributions of pressure, relative volume, velocity and mass fraction in chemical reaction zone are obtained, and comparisons are made with the exact solutions. Figure 1(a)-(d) gives the results where the mesh sizes of mesh 1-4 respectively are $\Delta r=1 / 1000,1 / 2000,1 / 5000,1 / 10000 \mathrm{~cm}$. At the same time, the relation of the Chapman-Jouguet velocity and Von Neumann pressure to the mesh sizes is given in Figure 2(a)-(b). From Figure 1, the shock front of detonation wave is well resolved, and the spurious oscillation does not appear in the vicinity of the shock discontinuity. From Figures 1 and 2, when the mesh size is less than $1 / 5000 \mathrm{~cm}$ (about 50 meshes in the reaction zone), the calculating solutions agree well with the exact solutions.

\section{Main conclusions}

1) This paper presents the relaxation method for numerically simulating the detonation in condensed explosives, and a temporal-spatial fifth-order precision scheme is utilized to discretize the relaxation equations, which does not require solving Riemann problem and calculating the Jacobian matrix of nonlinear flux and splitting the reaction source term. 


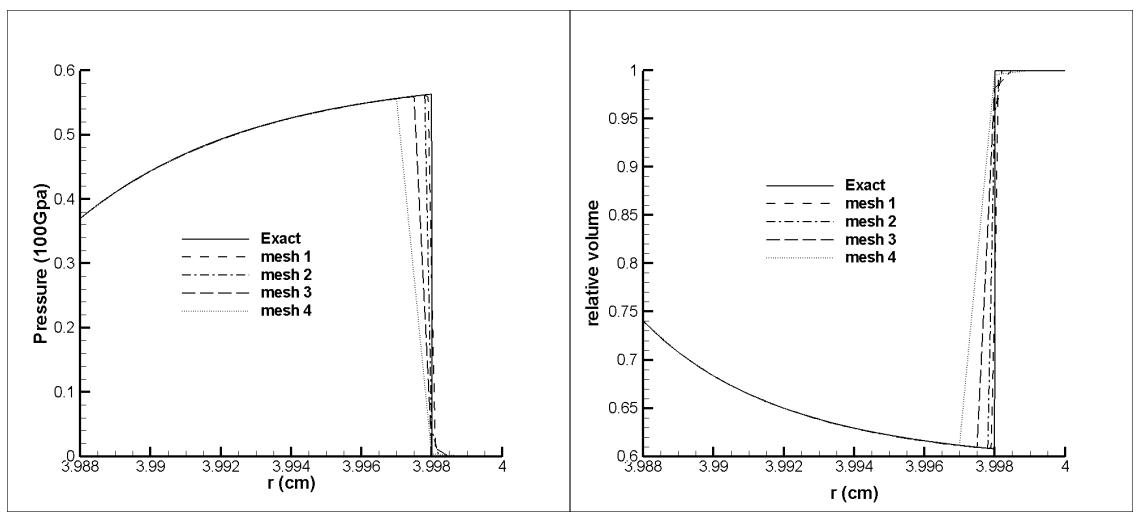

(a) pressure

(b) relative volume

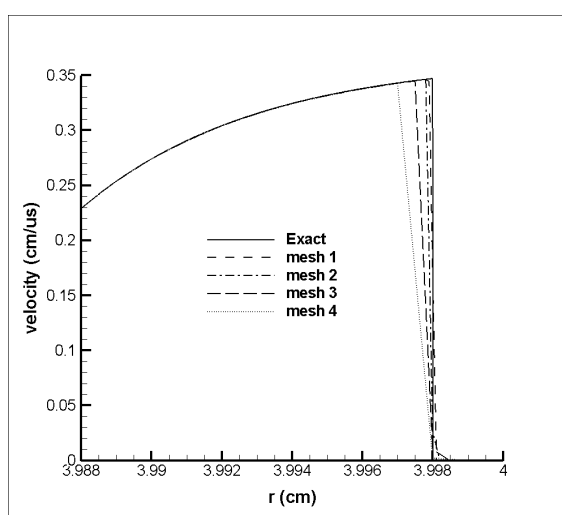

(c) velocity

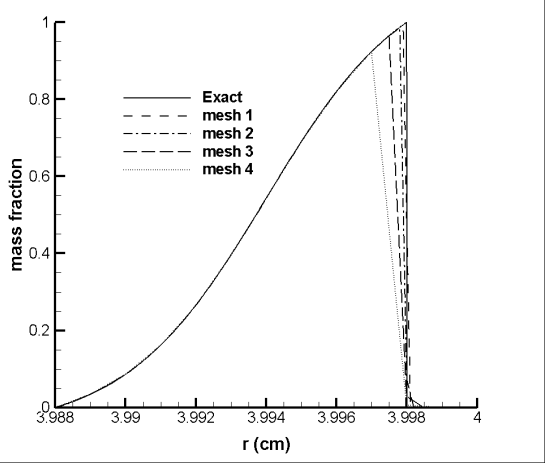

(d) mass fraction

Figure 1: The physical variables in chemical reaction zone of PBX-9404.

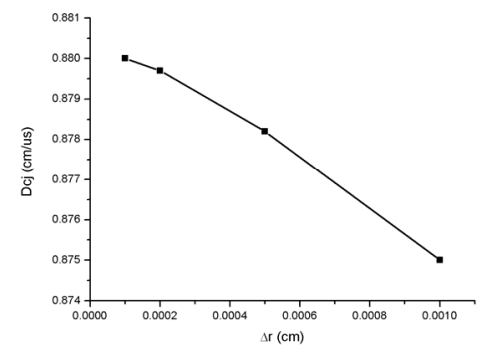

(a) CJ velocity

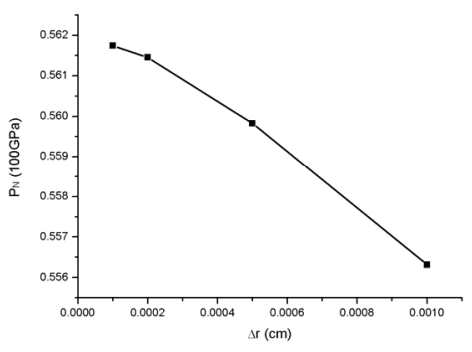

(b) Von Neumann pressure

Figure 2: The relation of the CJ velocity and Von Neumann pressure to the mesh sizes in PBX-9404. 
2) The calculating results for the steady structure of one-dimensional planar detonation wave in PBX-9404 demonstrate the high precision and high resolution of the present method.

3) The present method will be generalized to two-dimensional problems for condensed explosives.

\section{Acknowledgement}

Sponsored by the National Nature Science Foundation of China (11272064).

\section{References}

[1] C.L. Mader, Numerical modeling of explosives and propellants, $2^{\text {nd }}$ edition, CRC Press, New York, 1998.

[2] W.D. Henshaw and D.W. Schwedeman, An adaptive numerical scheme for high-speed reactive flow on overlapping grids, Journal of Computational Physics, 2003, 19(2): 420-447.

[3] A.K. Kapila, D.W. Schwedeman and J.B. Bdzil, A study of detonation diffraction in the Ignition-and-Growth Model, Combustion Theory and Modeling, 2007(11): 781-822.

[4] J.W. Banks, D.W. Schwedeman and A.K. Kapila, A study of detonation propagation and diffraction with compliant confinement, UCRL-JRNL233735, 2007.

[5] D.W. Schwedeman, A.K. Kapila and W.D. Henshaw, A study of detonation diffraction and failure for a model of compressible reactive flow, UCRL-JRNL-M43735, 2010.

[6] H.C. Yee, D.V. Kotov and B. Sjogreen, Numerical dissipation and wrong propagation speed of discontinuities for stiff source terms, Proceedings of ICCFD, Hawaii, 2011.

[7] H.C. Yee, D.V. Kotov and Chi-Wang Shu, Spurious behavior of shockcapturing methods: problems containing stiff source terms and discontinuities, Proceedings of ICCFD7, 2012.

[8] Sun Chengwei, Applied Detonation Physics, Defense Industry Press, Beijing, 2000.12 (in Chinese).

[9] W. Hundsdorfer and S.J. Ruuth, IMEX extensions of linear multistep methods with general monotonicity and boundedness properties, Journal of Computational Physics, 2007(225): 2016-2042.

[10] Liu T P, Hyperbolic conservation laws with relaxation, Comm. Math. Phys, 1987(108): 153-175.

[11] Jin S and Xin Z. The relaxation schemes for systems of conservation laws in arbitrary space dimensions, Communications of Pure and Applied Mathematics, 1995(48): 235-276.

[12] A. Chalabi, Convergence of relaxation schemes for hyperbolic conservation laws with stiff source, Mathematics of Computation, 1999(68): 955-970. 
[13] T. Tang, Convergence of MUSCL relaxing scheme to the relaxed scheme for conservation laws with stiff source terms, Journal of Scientific Computing, 2000, 15(2): 173-195.

[14] M.K. Banda and M. Seaid, A class of the relaxation schemes for 2D Euler systems of gas dynamics, ICCS 2002, LNCS 2329, 930-939.

[15] A.I. Delis and Th. Katsaounis, Numerical solution of the two-dimensional shallow water equations by the application of relaxation methods, Applied Mathematical Modelling, 2005(29): 754-783.

[16] WANG Ping, Numerical study of dynamics phase transitions in shock tube, Applied Mathematics and Mechanics, 2007, 28(7): 824-832 (in Chinese).

[17] S. B. Krishnamurthy and M. G. Gerritsen, A variable relaxation scheme for multiphase, multicomponent flow, Transaction Porous Medias, 2008(71): 345-377.

[18] M. K. Banda, Non-oscillatory relaxation schemes for one-dimensional ideal magnetohydrodynamic equations, Nonlinear Analysis: Real World Applications, 2009(10): 3345-3352.

[19] A.K. Henrick, T.D. Aslam, J.M. Powers, Mapped weighted essentially non-oscillatory schemes: Achieving optimal order near critical points, Journal of Computational Physics, 2005(207): 542-567. 